Nathaniel W. Yang, MD

Department of Otorhinolaryngology

College of Medicine - Philippine General Hospital University of the Philippines Manila

Philippine National Ear Institute National Institutes of Health University of the Philippines Manila

Department of Otolaryngology Head and Neck Surgery

Far Eastern University-Nicanor Reyes Memorial Foundation Institute of Medicine
Correspondence: Dr. Nathaniel W. Yang Department of Otorhinolaryngology Ward 10, Philippine General Hospital Taft Avenue, Ermita, Manila 1000 Philippines

Phone: (632) 5264360

Fax: (632) 5255444

Email: nwyang@gmx.net

Reprints will not be available from the author.

The author declared that this represents original material that is not being considered for publication or has not been published or accepted for publication elsewhere, in full or in part, in print or electronic media; that the manuscript has been read and approved by the author, that the requirements for authorship have been met by the author, and that the author believes that the manuscript represents honest work.

Disclosures: The author signed a disclosure that there are no financial or other (including personal) relationships, intellectual passion, political or religious beliefs, and institutional affiliations that might lead to a conflict of interest.

\section{Technique of Multi-Planar CT Image Reconstruction for the Evaluation of Superior Semicircular Canal Dehiscence Syndrome}

Superior semicircular canal dehiscence (SSCD) syndrome is an unusual cause of vertigo that was first identified by Minor in 1998. The patients initially described by Minor presented with vertigo, oscillopsia and/or dysequilibrium related to sound, changes in middle ear pressure and/or changes in intracranial pressure due to an absence of the bony layer that normally covers the superior semicircular canal. ' Subsequent clinical studies have shown that the condition may lead to a variety of vestibular and/or auditory symptoms that mimic other otologic disorders. These symptoms include autophony, ear blockage or fullness, conductive hearing loss, pulsatile tinnitus, dizziness or vertigo with head movements and general disequilibrium. ${ }^{2}$

In a patient with the appropriate symptoms, the diagnosis of superior semicircular canal dehiscence syndrome rests on the identification of a dehiscence in the bone overlying the semicircular canal on coronal high-resolution temporal bone computed tomographic scans (white arrowhead, Figure 1).

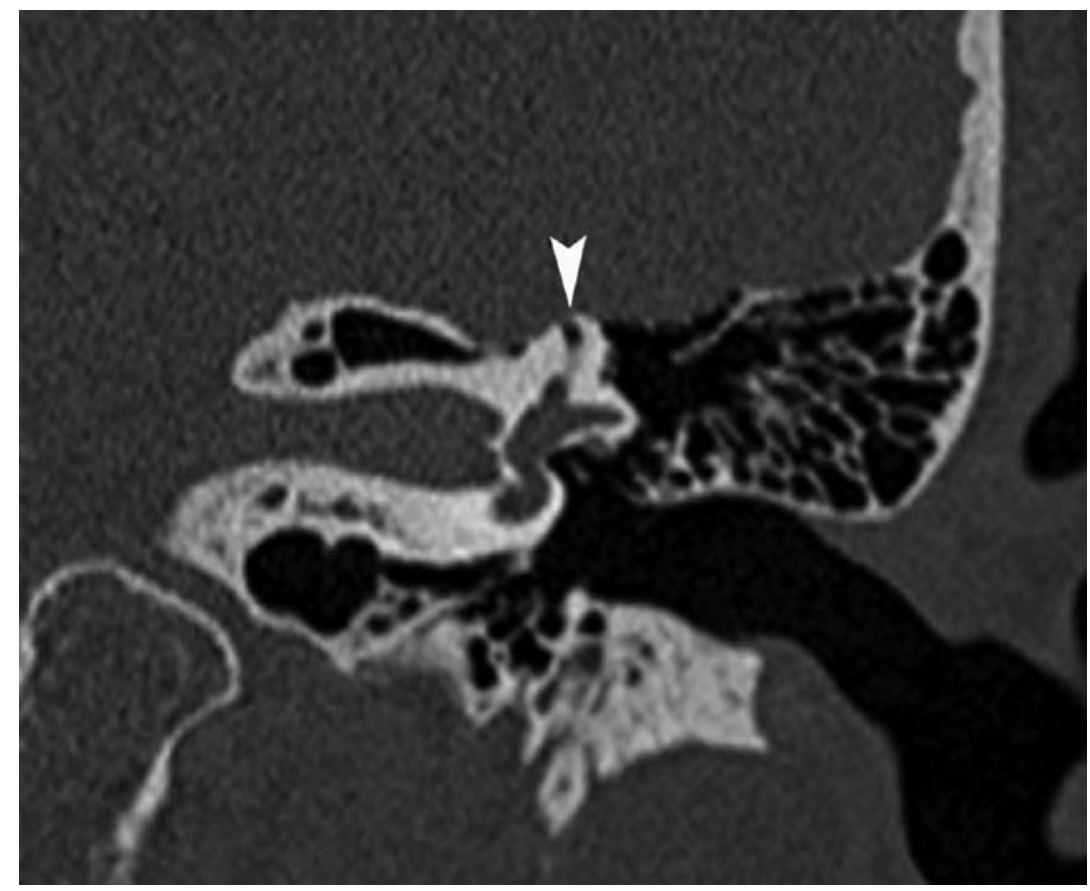

Figure 1. High-resolution CT scan of temporal bone, coronal view.

Note dehiscence in the bone overlying the semicircular canal (white arrowhead). 
It must be emphasized that due to the low specificity of images taken at $1.0-$ and $1.5-\mathrm{mm}$ collimation, current radiologic literature advocates the use of coronal reformatted images based on submillimeter $(0.4$ 0.6-mm) collimated axial scans. It must also be stressed that although reformatted coronal images are sufficient for the radiologic evaluation of SSCD in most cases, oblique reformatted images in the Stenver and Pöschl planes are necessary in equivocal cases. ${ }^{3}$ When operative management is indicated, these views are indispensable for proper surgical planning.

The images in oblique planes of reconstruction can be created on radiologic imaging software that allows multiplanar reconstruction (MPR) of the raw axial CT data set. This software allows the original data set to be simultaneously viewed in the standard sagittal, coronal and axial orthogonal planes of orientation (Figure 2) and manipulated into non-orthogonal or arbitary planes of orientation (oblique and doubleoblique).

The images in this article were made using syngo CT (version 2010B) software (Siemens AG, Berlin and München). This software has a set of toggle buttons in the 3D task window that allow manipulation of the primary image data set (Figure 3).

Button A (Free View Mode) - allows the reference lines in the three reconstructed views to be moved and rotated to achieve the desired image orientation. Moving the reference line in one view changes the position and orientation of the viewed image in the other two reconstructed views.

Button B (Hide Reference Lines) - removes the reference lines to have an unobstructed display of the reconstruction views

Button C (Orientation Control) - allows a 3D image to be rotated to the desired anatomic orientation. This function is not relevant to the task of identifying dehiscences in the semicircular canals

Button D (Rotate Images) - allows rotation of the image in three dimensions, independent of the reference lines

Button E (Zoom / Pan) - changes the magnification of the selected reconstruction view and allows the image to be moved in order to view the desired portion

Button F (Home Zoom / Pan) - returns all of the reconstructed views to their original orientation and magnification

The orthogonal axial image at the level of the superior semicircular canal is used as the primary reference image (Figure 4). The image has been zoomed in and panned using the Zoom/Pan function to center the image on the superior semicircular canal. With the Free View Mode activated, aligning the first reference line in the primary reference image to the long axis of the petrous bone and centered between the arms of the superior semicircular canal (white bar, Figure 4) creates a secondary reference image in the Stenver plane (Figure 5). Aligning the

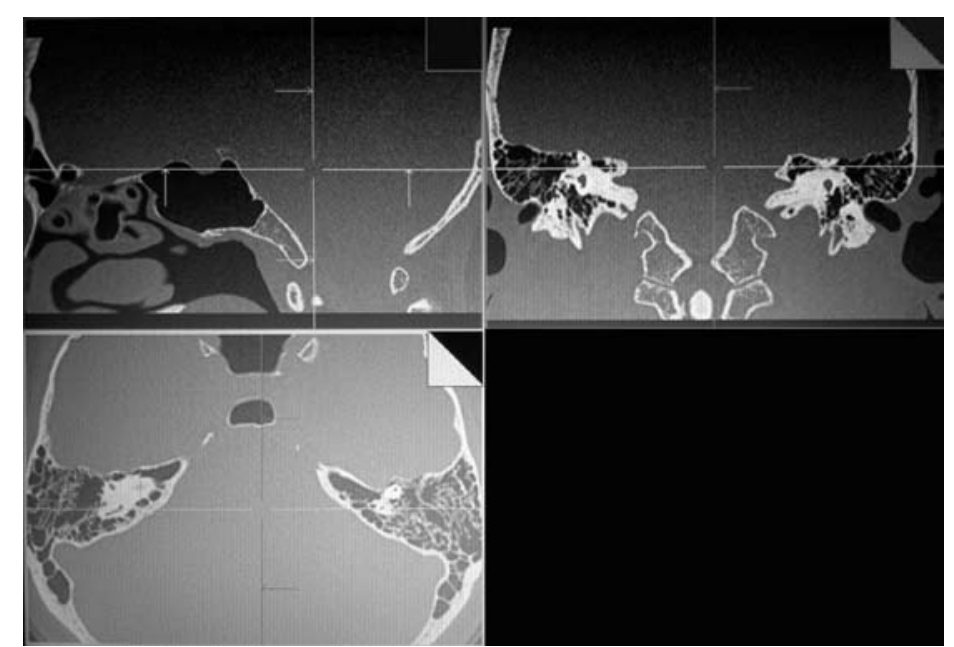

Figure 2. Multiplanar reconstruction in standard orthogonal planes of orientation (sagittal, coronal, axial) using syngo CT (version 2010B) software.

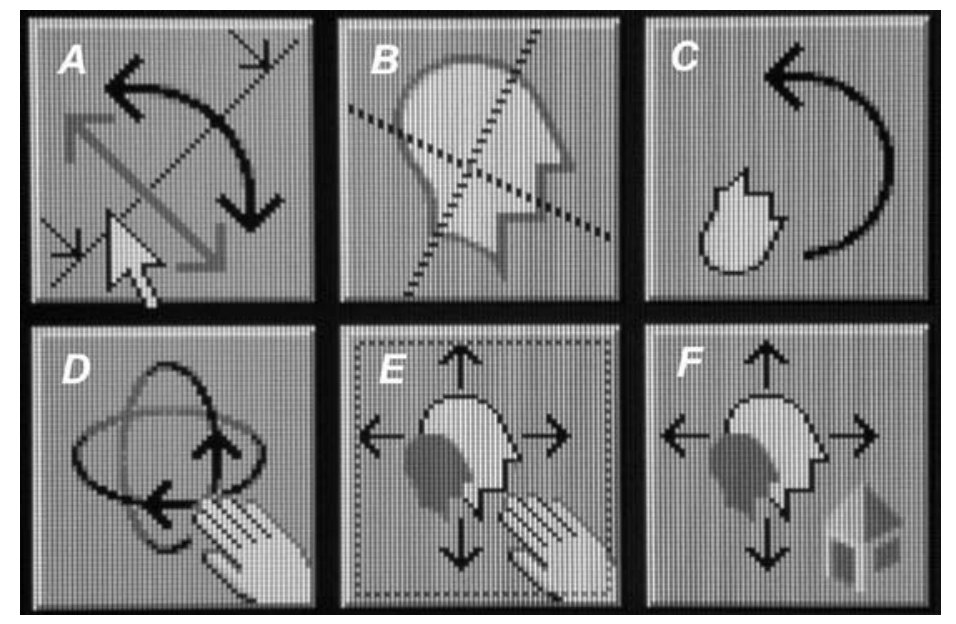

Figure 3. Toggle buttons in the 3D task window of syngo CT (version 2010B) software that allow manipulation of the primary image data set. The toggle buttons have been alphabetically labeled (A-F) for the purpose of identification in this article.

second reference line in the primary reference image to the short axis of the petrous bone and running through both arms of the superior semicircular canal (black arrow, Figure 4) creates the tertiary image in the Pöschl plane that allows visualization of the superior semicircular canal as a complete ring (Figure 6). As the superior semicircular canal may not be perfectly vertically oriented in the orthogonal axial reference image, adjustments in the vertical reference line of the secondary reference image in the Stenver plane to run along the long vertical axis of the superior semicircular canal are made (white bar, Figure 5). This will allow perfect visualization of the ring of the superior semicircular canal and any dehiscences of the overlying bone in the Pöschl plane (white arrowhead, Figure 6). 


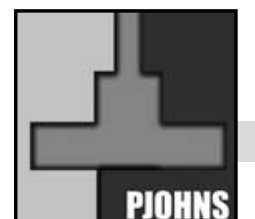

\section{PRACTICE PEARLS}

Philippine Journal Of Otolaryngology-Head And Neck Surgery

Vol. 26 No. 2 July - DeCEMBer 2011

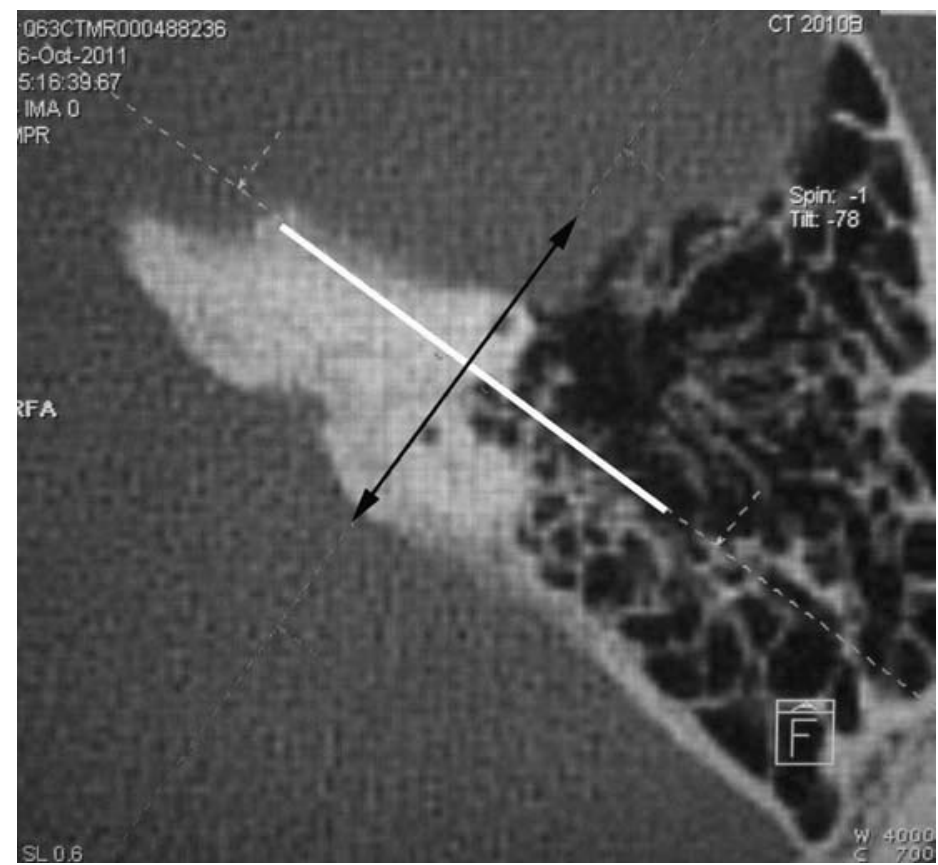

Figure 4. Primary reference image, orthogonal axial image, level of superior semicircular canal.

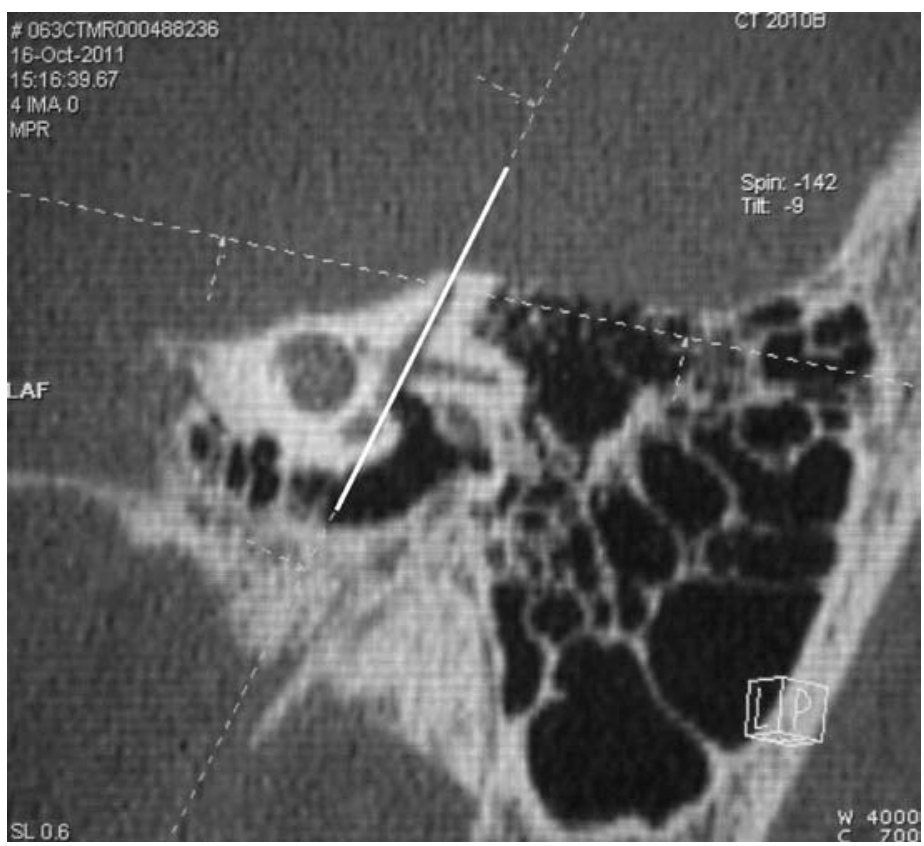

Figure 5. Secondary reference image, Stenver plane, created by aligning the first reference line in the primary reference image to the long axis of the petrous bone and centered between the arms of the semicircular canal.

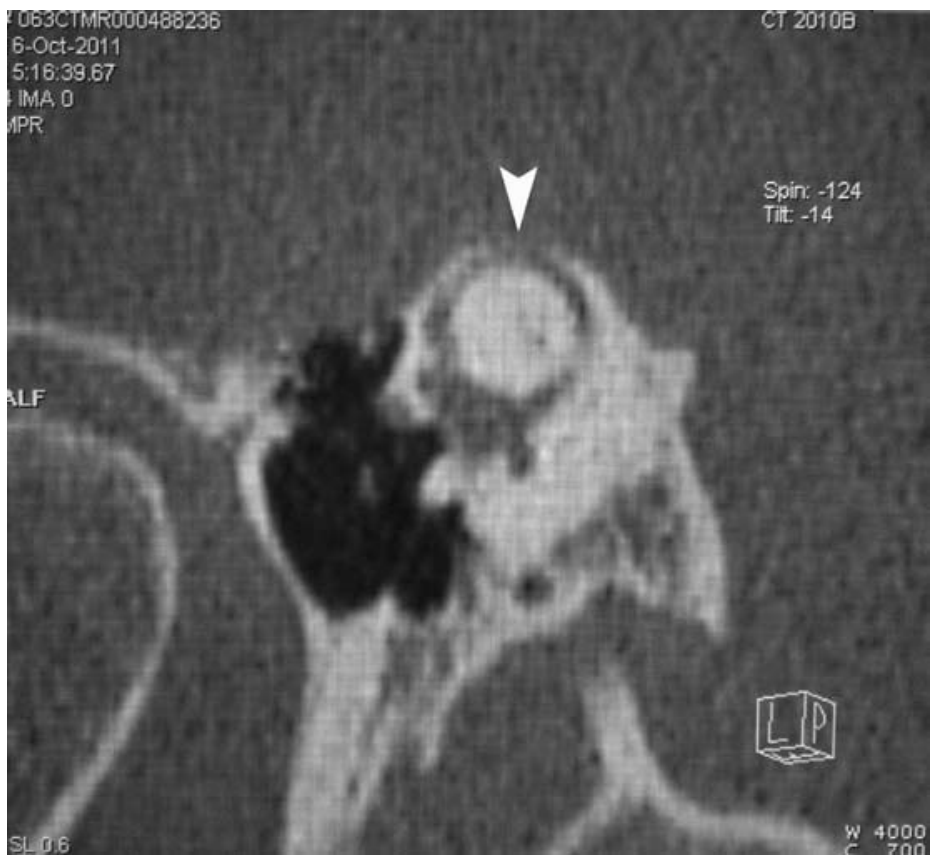

Figure 6. Tertiary image, Pöschl plane, allows visualization of the superior semicircular canal as a complete ring. Note dehiscence in the overlying bone (white arrowhead).

Imaging of the inner ear structures in non-orthogonal planes of orientation are not usually provided to the clinician by radiology centers in the Philippines. With a small investment in time and effort at the radiology workstation, it is possible for the clinician to view the inner ear structures especially the semicircular canals in their actual anatomic planes. This will allow the accurate diagnosis and management of less common but treatable causes of otologic symptoms.

\section{REFERENCES}

1. Minor LB, Solomon D, Zinreich JS, Zee DS. Sound- and/or pressure-induced vertigo due to bone dehiscence of the superior semicircular canal. Arch Otolaryngol Head Neck Surg. 1998 Mar 124(3):249-258.

2. Zhou G, Gopen Q, Poe DS. Clinical and diagnostic characterization of canal dehiscence syndrome: A great otologic mimicker. Otol Neurotol 2007 Oct; 28(7):920-926.

3. Branstetter BF 4th, Harrigal C, Escott EJ, Hirsch BE. Superior semicircular canal dehiscence: Oblique reformatted CT images for diagnosis. Radiology 2006 Mar; 238(3):938-942. 